\title{
Co-expression of podoplanin and fibroblast growth factor 1 predicts poor prognosis in patients with lung squamous cell carcinoma
}

\author{
JUAN LI ${ }^{1}$, HAN CHEN $^{2}$, XIAOQING LI ${ }^{3}$, LINLIN WANG ${ }^{1}$, AIQIN GAO ${ }^{1}$, PEI ZHANG ${ }^{4}$, WENLI LIN ${ }^{1}$, \\ WEI GAO ${ }^{5}$, DONG YANG ${ }^{1}$, XIAOSUN GUO ${ }^{6}$, JIE LIU ${ }^{1}$, QI DANG ${ }^{1}$ and YUPING SUN ${ }^{1}$ \\ ${ }^{1}$ Department of Oncology, Jinan Central Hospital, Shandong University, Jinan, Shandong 250013; \\ ${ }^{2}$ Soochow University College of Medicine, Suzhou, Jiangsu 215000; ${ }^{3}$ Zhejiang University School of Medicine, \\ Hangzhou, Zhejiang 310029; ${ }^{4}$ Department of Oncology, Nan Lou Division, Chinese PLA General Hospital, \\ Beijing 100853; ${ }^{5}$ Department of Pathology, Jinan Central Hospital; ${ }^{6}$ Department of Pathophysiology, \\ School of Medicine, Shandong University, Jinan, Shandong 250013, P.R. China
}

Received March 8, 2016; Accepted February 9, 2017

DOI: $10.3892 / \mathrm{mmr} .2017 .6830$

\begin{abstract}
Podoplanin and fibroblast growth factor (FGF) 1 have been detected more frequently in lung squamous cell carcinoma (SQCC) compared with lung adenocarcinoma. Furthermore, it has been previous demonstrated that FGF1 is located on the edge of tumor nests in certain lung SQCC sections, which resembles the characteristic expression pattern of podoplanin. Podoplanin and FGF1 have roles in lymphangiogenesis and angiogenesis. Based on their consistently specific expression in lung SQCC and similar localization patterns, the present study aimed to investigate whether the expression of podoplanin in tumor cells is correlated with FGF1 expression in lung SQCC and whether their co-expression has clinicopathological significance, particularly for lymphangiogenesis/angiogenesis. The correlation between podoplanin and FGF1 expression in tumor cells of 82 lung SQCC cases was investigated by immunohistochemical staining and the association between the co-expression of podoplanin and FGF1, and clinicopathological factors such as microvessel density (MVD), was examined in these samples. In addition, the prognostic value of co-expression of podoplanin and FGF1 in tumor cells was determined, and the regulation of FGF1 expression and angiogenesis by podoplanin was examined in vitro in a human lung SQCC cell line. Immunohistochemical analysis demonstrated that there was a significant correlation between podoplanin and FGF1 expression in lung SQCC tumor cells
\end{abstract}

Correspondence to: Professor Yuping Sun or Dr Qi Dang, Department of Oncology, Jinan Central Hospital, Shandong University, 105 Jie Fang Road, Jinan, Shandong 250013, P.R. China

E-mail: 13370582181@163.com

E-mail: dangqi123456@126.com

Key words: podoplanin, fibroblast growth factor 1, co-expression, angiogenesis, prognosis, lung squamous cell carcinoma
$(\mathrm{R}=0.591 ; \mathrm{P}<0.0001)$. Co-expression of podoplanin and FGF1 was significantly associated with larger primary tumor size, advanced TNM stage and higher intratumoral MVD. Survival analysis demonstrated that cases with podoplanin and FGF1 double-positive staining had a significantly lower survival rate compared with cases with double-negative staining. In vitro experiments revealed that podoplanin regulated FGF1 expression and affected tube formation of human umbilical vein endothelial cells. Combined, the results demonstrated that podoplanin was co-expressed with FGF1 in lung SQCC and this co-expression was correlated with poor prognosis.

\section{Introduction}

Lung cancer is the leading cause of cancer-associated death worldwide, $\sim 85 \%$ of which is non-small cell lung cancer (NSCLC) (1). The two major histological subtypes of NSCLC are adenocarcinoma (30-50\% of cases) and squamous cell carcinoma (SQCC; $\sim 30 \%$ of cases) (2). There have been advances in molecularly-targeted agents for the treatment of lung adenocarcinoma $(3,4)$, however, progress in the treatment of lung SQCC has been limited. An increasing number of studies have supported the notion that adenocarcinoma and SQCC are not a homogeneous group of tumors and that they should be examined separately to identify potential molecular targets $(5,6)$. Thus, the identification of novel, potential therapeutic targets for lung SQCC is required to improve treatment of this type of cancer.

Podoplanin is a mucin-like transmembrane glycoprotein that is highly and specifically expressed in lymphatic endothelial cells (7). Multiple studies have demonstrated that podoplanin is upregulated in a number of cancers, including NSCLC (8) and it has been identified as a candidate cancer stem cell marker (9). Notably, podoplanin is frequently detected in lung SQCC tumor cells, however, it is rarely observed in lung adenocarcinoma tumor cells (8).

Our previous study demonstrated that the expression level of fibroblast growth factor (FGF) 1 was also significantly 
higher in lung SQCC compared with lung adenocarcinoma, and FGF1 was associated with poor prognosis in lung SQCC, which was not the case in lung adenocarcinoma (10). Furthermore, our previous study indicated that FGF1 was predominantly located on the edge of tumor nests in certain lung SQCC sections, which resembles the characteristic expression pattern of podoplanin (11). Based on the consistently specific expression of podoplanin and FGF1 in lung SQCC tissues and their similar location pattern, the present study investigated whether tumor-cell expression of podoplanin is correlated with that of FGF1, and whether co-expression is associated with clinicopathological factors and prognosis in lung SQCC. To the best of our knowledge, the correlation between podoplanin and FGF1 expression and the clinicopathological significance of their co-expression has not been previously investigated.

As a potent mitogenic growth factor, FGF1 promotes the proliferation, migration and survival of vascular (12) and lymphatic endothelial cells (13), which are essential for angiogenesis and lymphangiogenesis, respectively. Similarly, podoplanin has certain roles in tumor lymphangiogenesis and angiogenesis. Researchers have previously demonstrated that podoplanin regulates the expression of vascular endothelial growth factor C (VEGFC) (14) and endothelin-1 (15), which affects lymphangiogenesis. Pula et al (16) reported that podoplanin expression in cancer-associated fibroblasts (CAFs) was positively correlated with intratumoral microvessel density (MVD), which suggested a role for podoplanin in angiogenesis. However, clinical correlations between podoplanin expression and lymphangiogenesis/angiogenesis in human lung SQCC tissues have not previously been reported. Therefore, the present study aimed to investigate whether podoplanin may regulate the expression of FGF1 to influence tumor lymphangiogenesis and/or angiogenesis in lung SQCC.

The current study examined the correlation between podoplanin and FGF1 expression in cancer cells of 82 lung SQCC cases (stage I-IV) by immunohistochemical (IHC) staining and investigated the association between podoplanin/FGF1 co-expression and clinicopathological factors, such as MVD in these samples. In addition, the prognostic value of co-expression of podoplanin and FGF1 in lung SQCC tumor cells was examined, and the potential regulation of FGF1 expression and angiogenesis by podoplanin in vitro in a human lung SQCC cell line was investigated.

\section{Materials and methods}

Patients. Tumor specimens were obtained from 82 patients with primary lung SQCC who underwent surgery at the Jinan Central Hospital of Shandong University (Jinan, China) between January 2006 and May 2009. Patients had not received radiation therapy or chemotherapy prior to biopsy or surgical resection. Written informed consent was obtained from each patient. The study was approved by the Institutional Review Board of Jinan Central Hospital of Shandong University. Patients included 74 men and 8 women with a median age of 62 (range, 41-82) years at the time of diagnosis. The tumor, node, metastasis (TNM) classification was performed according to the Union for International Cancer Control 7th edition staging system for NSCLC (17). Patients were followed up for a median follow-up period of 19.5 months (range, 3-60 months) following surgery.

Cell lines and cell culture. NCI-H226 human lung SQCC cell line and human umbilical vein endothelial cells (HUVECs) were obtained from American Type Culture Collection (Manassas, VA, USA). NCI-H226 SQCC cells were cultured in RPMI-1640 medium supplemented with $10 \%$ fetal bovine serum (both from Hyclone; GE Healthcare Life Sciences, Logan, UT, USA) and $100 \mathrm{U} / \mathrm{ml}$ penicillin-streptomycin. HUVECs were cultured in endothelial cell medium (ScienCell Research Laboratories, Inc., Carlsbad, CA, USA). Cells were maintained at $37^{\circ} \mathrm{C}$ with $5 \% \mathrm{CO}_{2}$ in a humidified incubator.

Gene silencing. Small interfering RNAs (siRNAs; 21-nucleotides-long) targeting podoplanin (siRNA-1 and siRNA-2; Shanghai GenePharma Co., Ltd., Shanghai, China) were transfected into NCI-H226 cells using Lipofectamine ${ }^{\circledR} 2000$ transfection reagent (Invitrogen; Thermo Fisher Scientific, Inc., Waltham, MA, USA) according to the manufacturer's instructions. Scrambled siRNA was used as a negative control (NC). siRNA sequences were as follows: siRNA-1, 5'-GCG CAAGAACAAAGUCCA ATT-3'; siRNA-2, 5'-GACCCU GGUUGGAAUCAUATT-3'; and NC, 5'-UUCUCCGAACGU GUCACGUTT-3'. Transfected cells were harvested after 48 and $72 \mathrm{~h}$, the effect on podoplanin expression was assessed and cells were subsequently used for further experiments.

Reverse transcription-quantitative polymerase chain reaction $(R T-q P C R)$. Total RNA was isolated from cultured cells using TRIzol reagent (Invitrogen; Thermo Fisher Scientific, Inc.) and reverse transcription was performed using the RevertAid First Strand cDNA Synthesis kit (Thermo Fisher Scientific, Inc.). qPCR was performed using Maxima SYBR-Green qPCR Master Mix (Thermo Fisher Scientific, Inc.) on a CFX96 ${ }^{\mathrm{TM}}$ Real-Time PCR Detection System (Bio-Rad Laboratories, Inc., Hercules, CA, USA). The following primers were used for qPCR: Human podoplanin, 5'-GATGGAGACACACAGACA ACAGT-3' (forward) and 5'-TTTTCGCATAACCACAAC GAT-3' (reverse); human FGF1, 5'-GTGGATGGGACAAGG GACAG-3' (forward) and 5'-GGCAGGGGGAGAAACAAG AT-3' (reverse); and human GAPDH 5'-AGAAGGCTGGGG CTCATTTG-3' (forward) and 5'-AGGGGCCATCCACAG TCTTC-3' (reverse). The reaction conditions consisted of $50^{\circ} \mathrm{C}$ for $2 \mathrm{~min}$ and $94^{\circ} \mathrm{C}$ for $10 \mathrm{~min}$ (initial denaturation), followed by 40 cycles of $94^{\circ} \mathrm{C}$ for $15 \mathrm{sec}$ and $60^{\circ} \mathrm{C}$ for $60 \mathrm{sec}$. This was followed by melting curve analysis to verify the specificity and identity of the PCR product. Amplification results for qPCR were calculated using the $2^{-\Delta \Delta \mathrm{Cq}}$ method (18) and expression was normalized to that of GAPDH. The experiments were repeated at least three times.

Western blot analysis. Cells were lysed with lysis buffer (Beyotime Institute of Biotechnology, Haimen, China) containing protease inhibitor phenylmethane sulfonyl fluoride (Beyotime Institute Biotechnology), and the supernatant of the lysed cells was recovered. Protein concentrations were quantified using a bicinchoninic acid protein assay. Protein samples (50 $\mu \mathrm{g}$ per lane) were separated by $10 \%$ SDS-PAGE and transferred onto polyvinylidene difluoride membranes. 
Non-specific binding was blocked with $5 \%$ skim milk in TBS containing $0.1 \%$ Tween-20 for $1 \mathrm{~h}$ at room temperature. Subsequently, blotted membranes were incubated overnight at $4^{\circ} \mathrm{C}$ with specific primary antibodies. The following primary antibodies were used: Monoclonal rabbit anti-human podoplanin antibody (1:1,000; cat. no. 9047; Cell Signaling Technology, Inc., Danvers, MA, USA); monoclonal mouse anti-human FGF1 antibody (1:500; cat. no. H00002246-M02; Abnova Corporation, Taipei, Taiwan); and monoclonal mouse anti-human $\alpha$-tubulin antibody (1:5,000; cat. no. 66031-1-Ig; ProteinTech Group, Inc., Chicago, IL, USA) as a loading control. Detection was performed using horseradish peroxidase-conjugated secondary antibodies (1:5,000; cat. nos. SA00001-1 and SA00001-2; ProteinTech Group, Inc.). Finally, blots were immersed in ECL detection reagent (EMD Millipore, Billerica, MA, USA) and exposed to a ChemiDoc ${ }^{\mathrm{TM}}$ XRS+ system (Bio-Rad Laboratories, Inc.).

Preparation and concentration of culture supernatants. To prepare culture supernatants, $2.5 \times 10^{5}$ NCI-H226 cells were plated in 6-well plates and transfected with podoplanin siRNAs as described above. Culture medium was replaced with serum-free medium at $12 \mathrm{~h}$ after transfection and cells were cultured for a further $60 \mathrm{~h}$. Subsequently, supernatants were harvested and centrifuged at $1,000 \mathrm{x} \mathrm{g}$ for $10 \mathrm{~min}$. For western blot analysis, culture supernatants were concentrated with Amicon Ultra-0.5 Centrifugal Filter Unit with Ultracel-3 membrane (EMD Millipore) according to the manufacturer's instructions.

Capillary tube formation assay. For investigation of capillary tube formation, 96-well plates were coated with $60 \mu \mathrm{l}$ growth factor-reduced Matrigel (BD Biosciences, Franklin Lakes, NJ, USA) and incubated at $37^{\circ} \mathrm{C}$ for $1 \mathrm{~h}$ to allow gelling. Tumor cell culture supernatants were prepared as described above. HUVECs were resuspended using supernatants collected from cultured tumor cells and seeded on Matrigel-coated 48-well plates at a density of $2 \times 10^{4}$ cells/well. HUVECs were incubated for $8 \mathrm{~h}$. The branch points of the formed tubes, which represent the degree of angiogenesis in vitro, were imaged under a light microscope and quantified in 10 microscopic fields.

IHC analysis of tumor specimens. Formalin-fixed, paraffinembedded tissue sections ( $4 \mu \mathrm{m}$-thick) were deparaffinized and rehydrated. For FGF1, antigen retrieval was performed by incubating specimens for $5 \mathrm{~min}$ in $1 \% \mathrm{SDS}$ in TBS at room temperature. For podoplanin, CD34 and D2-40, antigen retrieval was performed by heating specimens in a microwave oven for $10 \mathrm{~min}$ with $10 \mathrm{mmol} / \mathrm{l}$ sodium citrate ( $\mathrm{pH}$ 6.0). Endogenous peroxidase activity was blocked with $0.3 \%$ hydrogen peroxide for $10 \mathrm{~min}$. Sections were blocked with 5\% normal goat serum (cat. no. SP Kit-B1; Fuzhou Maixin Biotech. Co., Ltd., Fuzhou, China) for $20 \mathrm{~min}$ at room temperature and were subsequently incubated overnight at $4^{\circ} \mathrm{C}$ with primary antibodies; monoclonal rabbit anti-human podoplanin antibody (1:600; cat. no. 9047; Cell Signaling Technology), monoclonal mouse anti-human FGF1 antibody (1:300; cat. no. H00002246-M02; Abnova Corporation), monoclonal mouse anti-human CD34 antibody (1:100; cat. no. Kit-0004; Fuzhou Maixin Biotech. Co., Ltd.) and monoclonal mouse anti-human D2-40 antibody (1:100; cat. no. MAB-0567, Fuzhou Maixin Biotech Co., Ltd.). To detect primary antibody binding, sections were incubated with Elivision super Polymer horseradish peroxidase (Mouse/Rabbit) IHC kit (cat. no. Kit-9922, Fuzhou Maixin Biotech Co., Ltd.) according to the manufacturer's protocol. Sections were visualized with 3,3'-diaminobenzidine solution and counterstained with hematoxylin. Human tonsil tissues and lymphatic vessels in tumors were selected as positive controls for FGF1 and podoplanin, respectively. Negative controls were prepared using normal mouse and rabbit IgG instead of the primary antibody. The expression was quantified simultaneously by two independent observers who were blind to the details of the patients. The percentage of stained cells was recorded at x400 magnification in $\leq 5$ random fields. Evaluation of the cell staining reaction was performed using the immunoreactive score (IRS) as follows: IRS=staining intensity (SI) x percentage of positive cells (PP), as previously described (19). SI was assigned as 0 , negative; 1 , weak; 2 , moderate; and 3 , strong. PP was defined as 0 , negative; $1,1-10 \%$ positive cells; $2,11-50 \%$ positive cells; $3,51-80 \%$ positive cells; and $4,>80 \%$ positive cells. For statistical analyses, cases with scores 0-3 were defined as negative and all others were considered positive.

MVD and lymphatic MVD (LMVD) were assessed according to a modification of Weidner's method (20). MVD was detected by CD34 antibody and LMVD was detected by D2-40 antibody. The immunostained sections were viewed using light-microscopy at low magnification (x40) and the areas of tissue with the greatest number of distinctly highlighted microvessels (hotspots) were selected. MVD and LMVD were determined by counting all immunostained vessels at a total magnification of $\mathrm{x} 400$ from 5 areas for each case. Determination of the staining reaction was strictly confined to the hotspots and the mean number of the vessels in each case was evaluated.

Statistical analysis. SPSS version 18.0 (SPSS, Inc., Chicago, IL, USA) was used for statistical analyses. Spearman correlation analysis was used to analyze the correlation between podoplanin and FGF1 expression. The association between co-expression of podoplanin and FGF1, and clinicopathological variables were analyzed using the $\chi^{2}$ test. Associations between podoplanin/FGF1 expression and LMVD/MVD were analyzed by a two-tailed Student's t-test. Comparisons of mRNA expression in NC and siRNA groups were performed by two-tailed Student t-test. Overall survival time was defined as the period from the date of surgery to the date of death from any cause or the last day of follow-up evaluation. Survival curves were produced using the Kaplan-Meier method and compared with log-rank test. For all tests, $\mathrm{P}<0.05$ was considered to indicate a statistically significant difference.

\section{Results}

Expression of podoplanin and FGF1 in human primary lung SQCC tissues. The expression of podoplanin and FGF1 was examined by IHC staining in 82 human lung SQCC specimens. Consistent with previous reports, expression of podoplanin was detected on the membrane and/or cytoplasm of tumor cells (Fig. 1A and B). Negative cases were determined by the 
A

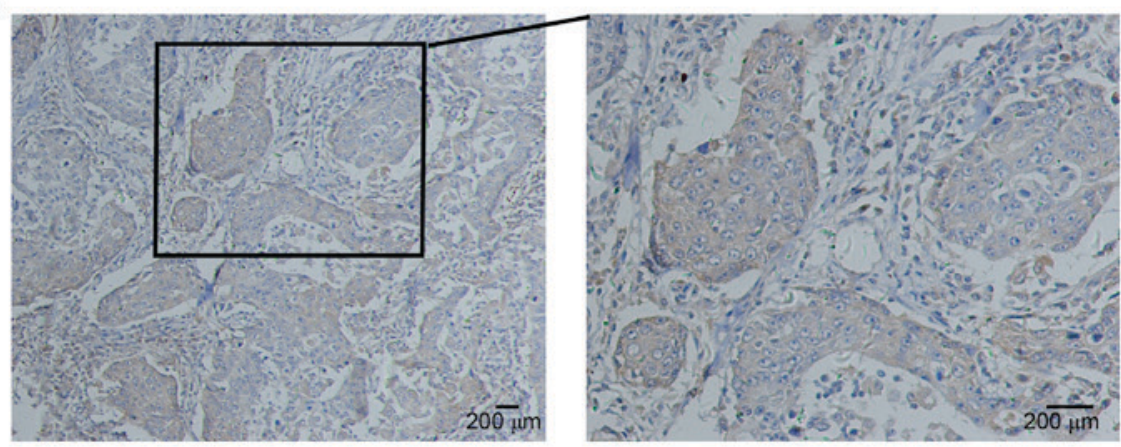

B

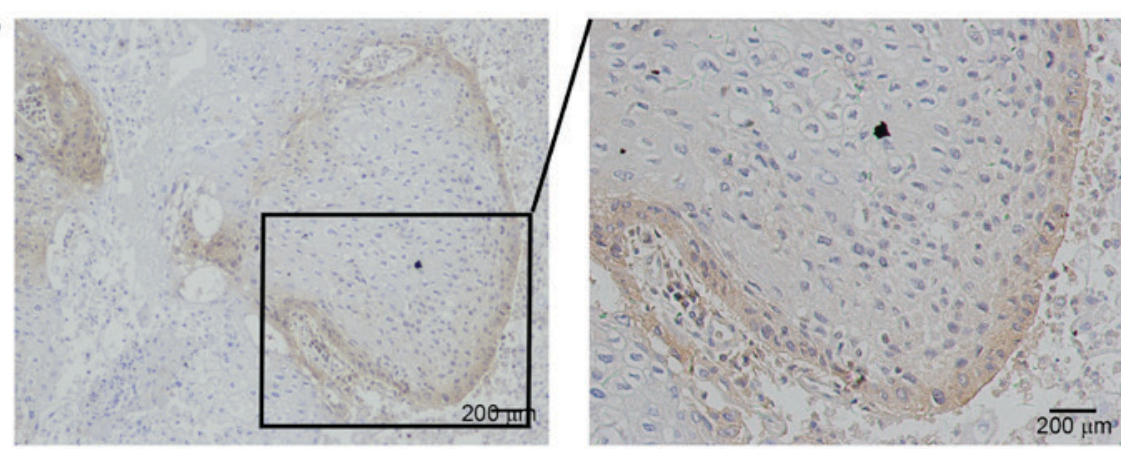

C

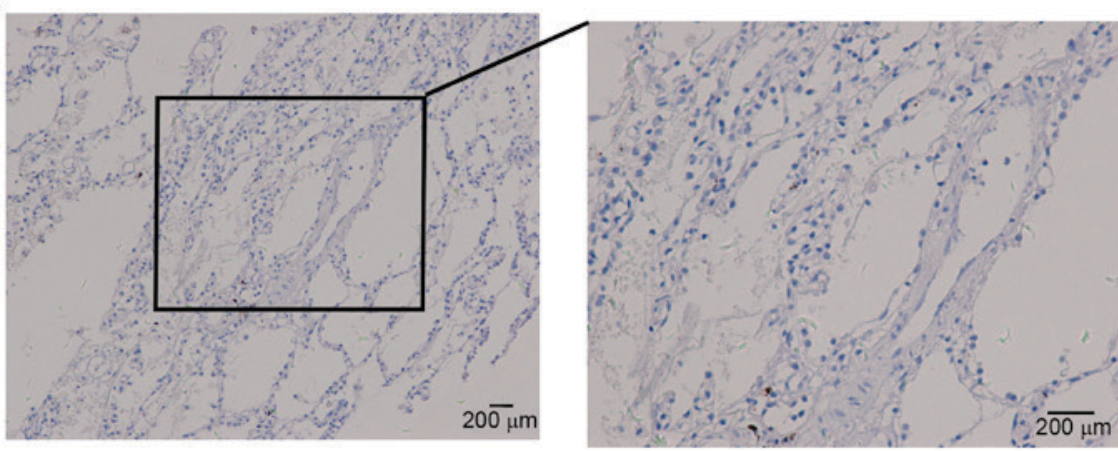

D

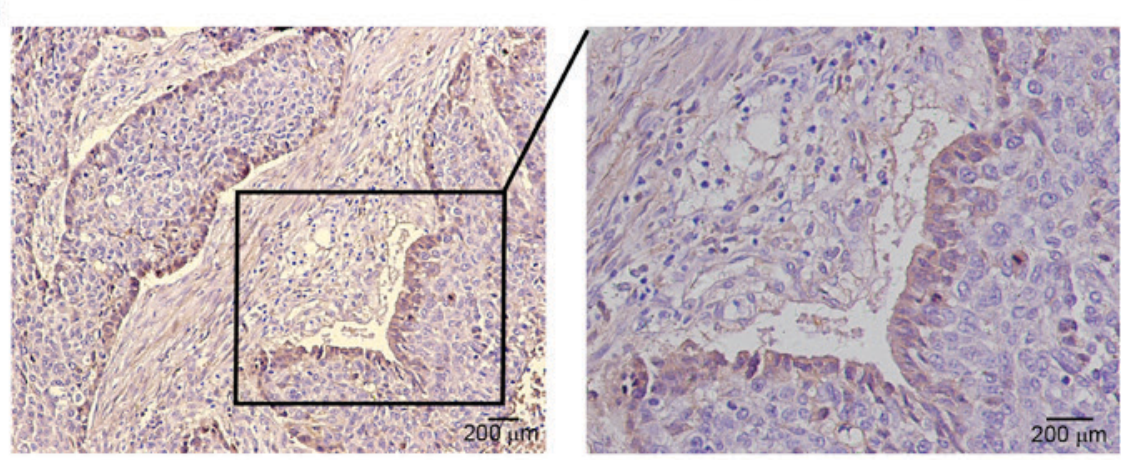

$\mathrm{E}$

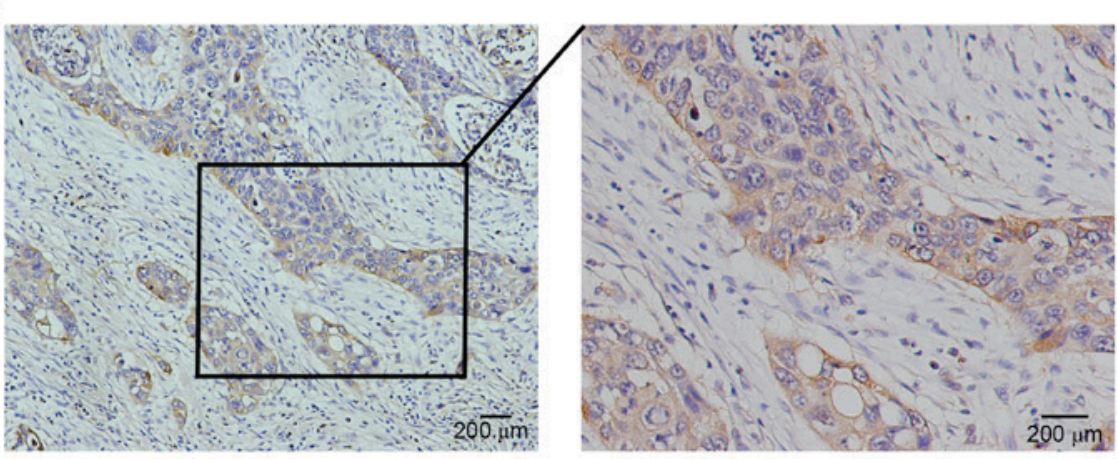

Figure 1. Expression of podoplanin and FGF1 in human primary lung squamous cell carcinoma tissues. (A) Diffuse expression of podoplanin in the entire cancer nest. (B) Peripheral expression of podoplanin in the cancer nest. (C) Negative expression of podoplanin in peritumoral normal lung tissue. (D) Peripheral expression of FGF1 in the cancer nest. (E) Diffuse expression of FGF1 in the entire cancer nest. Higher magnification of boxed areas are shown on the right. FGF1, fibroblast growth factor 1 . 

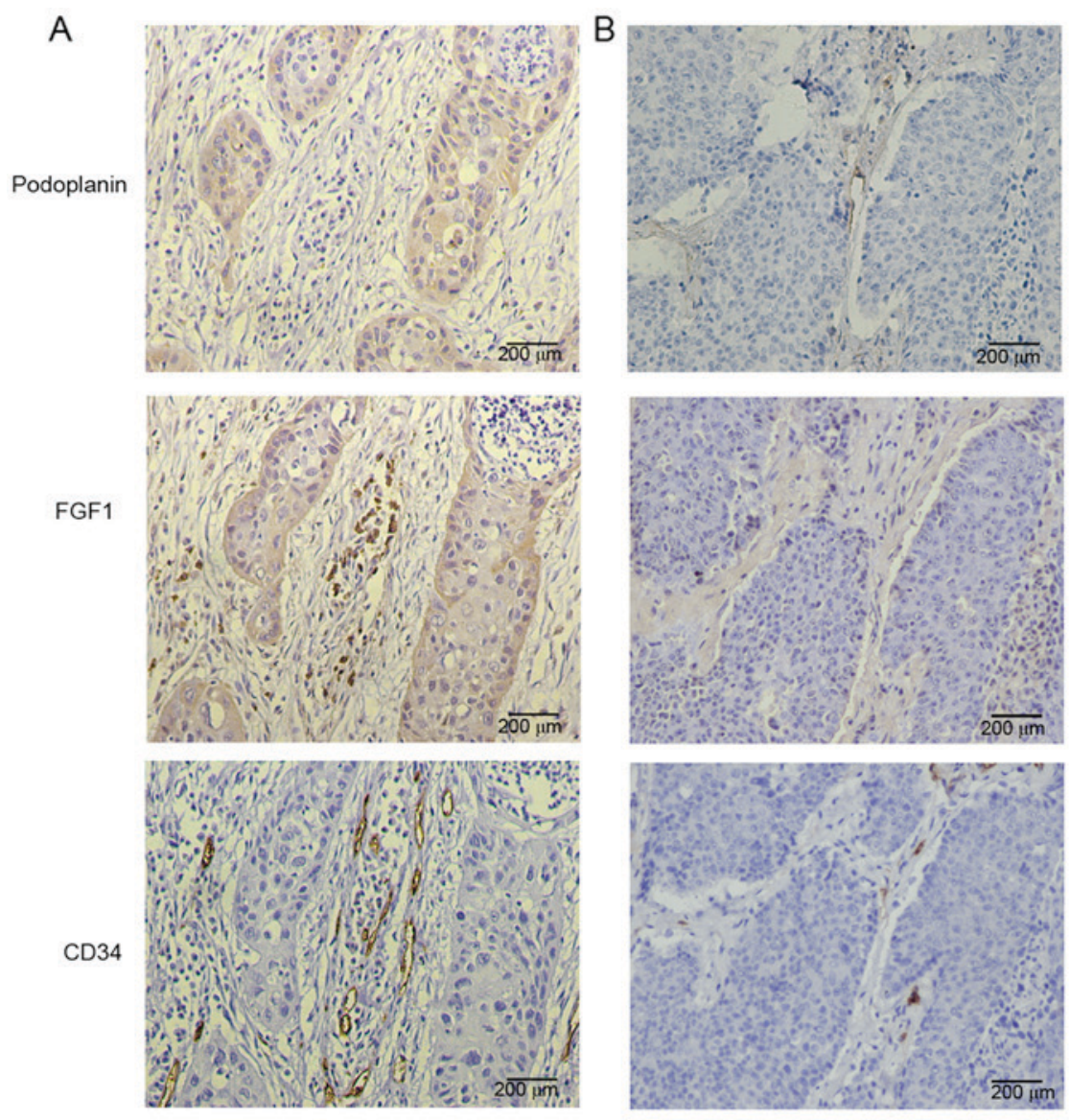

C

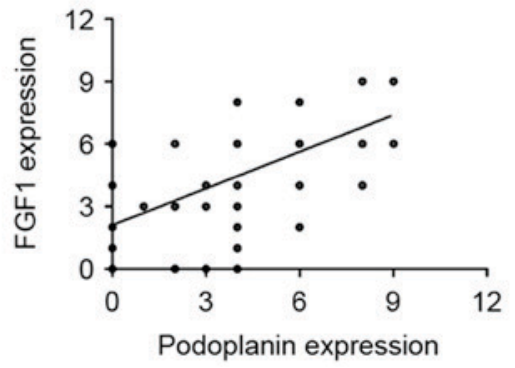

D

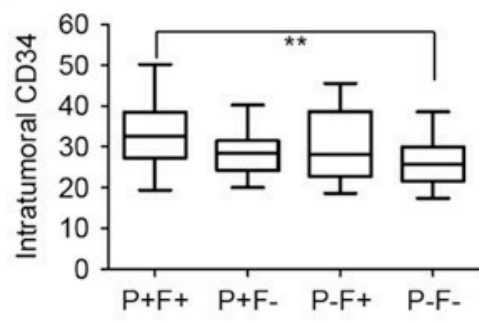

Figure 2. Consecutive sections of lung squamous cell carcinoma were stained for podoplanin, FGF1 and CD34. Representative images of sections of cancer tissue with (A) positive podoplanin expression and (B) negative podoplanin expression stained for podoplanin, FGF1 and CD34. (C) Significant correlation between podoplanin and FGF1 expression in tumor cells by Spearman correlation analysis ( R=0.591, $\mathrm{P}<0.0001$ ). (D) Comparison of intratumoral MVD among patients with $\mathrm{P}+\mathrm{F}+, \mathrm{P}+\mathrm{F}-, \mathrm{P}-\mathrm{F}+$ and $\mathrm{P}-\mathrm{F}-$. MVD in $\mathrm{P}+\mathrm{F}+$ group was significantly higher than that in $\mathrm{P}-\mathrm{F}-$ group. ${ }^{* *} \mathrm{P}<0.01$. FGF1, fibroblast growth factor 1 ; MVD, microvessel density; $\mathrm{P}+\mathrm{F}+$, podoplanin-positive/FGF1-positive; $\mathrm{P}+\mathrm{F}-$, podoplanin-positive/FGF1-negative; $\mathrm{P}-\mathrm{F}+$, podoplanin-negative/FGF1-positive; P-F-, podoplanin-negative/FGF1-negative.

staining of lymphatic vessels as an internal control. In addition, podoplanin expression was observed in stromal spindle cells that were morphologically identified as fibroblasts. There was negative or weak staining for podoplanin in peritumoral normal lung tissues (Fig. 1C). Positive expression of podoplanin (IRS >3) in tumor cells was detected in $73.17 \%(60 / 82)$ cases. Higher than previously reported (11), 38.3\% (23/60) of positive cases exhibited diffuse immunoreactions in almost the entire cancer nest (Fig. 1A). Other positive cases exhibited a peripheral expression pattern; expression was restricted to the outer cell layer of tumor nests (Fig. 1B).

Immunostaining of FGF1 in the majority of cases was cytoplasmic, while a few cases exhibited nuclear and/or perinuclear staining in cancer cells. Positive expression of
FGF1 in tumor cells was detected in $70.73 \%$ (58/82) lung SQCC samples. Notably, 34.48\% (20/58) cases with positive FGF1 expression exhibited a non-homogeneous peripheral expression pattern (Fig. 1D), which is similar to the expression pattern of podoplanin. The remaining positive cases exhibited diffuse expression of FGF1 in the entire cancer nest (Fig. 1E). In addition, there was frequent expression of FGF1 in lung SQCC tumor stromal cells.

Co-expression of podoplanin and FGF1 in primary lung SQCC tissues, and the association between co-expression and clinicopathological factors. Podoplanin and FGF1 were demonstrated to be co-expressed in lung SQCC tumor cells (Fig. 2A and B), and there was a significant, positive 
Table I. Association between podoplanin and FGF1 co-expression and clinicopathological factors in lung squamous cell carcinoma patients.

\begin{tabular}{|c|c|c|c|c|c|c|c|}
\hline Variables & $\begin{array}{c}\mathrm{P}+\mathrm{F}+ \\
\mathrm{n}\end{array}$ & P-value & $\begin{array}{c}\mathrm{P}+\mathrm{F}- \\
\mathrm{n}\end{array}$ & P-value & $\begin{array}{c}\mathrm{P}-\mathrm{F}+ \\
\mathrm{n}\end{array}$ & P-value & $\begin{array}{c}\text { P-F- } \\
n\end{array}$ \\
\hline \multicolumn{8}{|l|}{ Age, years } \\
\hline$\leq 60$ & 22 & 0.299 & 4 & 0.673 & 3 & 1 & 4 \\
\hline$>60$ & 28 & & 6 & & 5 & & 10 \\
\hline \multicolumn{8}{|l|}{ Sex } \\
\hline Male & 44 & 0.976 & 9 & 1 & 8 & 1 & 13 \\
\hline Female & 6 & & 1 & & 0 & & 1 \\
\hline \multicolumn{8}{|c|}{ Smoking index, packs/year } \\
\hline$\leq 400$ & 17 & 0.568 & 1 & 0.615 & 1 & 1 & 3 \\
\hline$>400$ & 33 & & 9 & & 7 & & 11 \\
\hline \multicolumn{8}{|l|}{ Differentiation } \\
\hline Well/moderate & 26 & 0.545 & 5 & 1 & 5 & 0.659 & 6 \\
\hline Poor & 24 & & 5 & & 3 & & 8 \\
\hline \multicolumn{8}{|c|}{ Primary tumor size, $\mathrm{cm}$} \\
\hline$\leq 5$ & 24 & $0.042^{\mathrm{a}}$ & 7 & 0.665 & 5 & 0.624 & 11 \\
\hline$>5$ & 26 & & 3 & & 3 & & 3 \\
\hline \multicolumn{8}{|c|}{ Lymph node metastasis } \\
\hline Yes & 35 & 0.061 & 6 & 0.68 & 4 & 1 & 6 \\
\hline No & 15 & & 4 & & 4 & & 8 \\
\hline \multicolumn{8}{|c|}{ Vascular invasion } \\
\hline Yes & 9 & 0.201 & 0 & NA & 1 & 0.364 & 0 \\
\hline No & 41 & & 10 & & 7 & & 14 \\
\hline \multicolumn{8}{|c|}{ Pleural metastasis } \\
\hline Yes & 9 & 0.201 & 1 & 0.417 & 1 & 0.364 & 0 \\
\hline No & 41 & & 9 & & 7 & & 14 \\
\hline \multicolumn{8}{|l|}{ TNM stage } \\
\hline I-II & 28 & $0.042^{\mathrm{a}}$ & 7 & 0.615 & 6 & 0.602 & 12 \\
\hline III-IV & 22 & & 3 & & 2 & & 2 \\
\hline
\end{tabular}

All P-values are vs. the $\mathrm{P}$-F- group. ${ }^{\mathrm{a}} \mathrm{P}<0.05 . \mathrm{P}+\mathrm{F}+$, podoplanin-positive/FGF1-positive cases; $\mathrm{P}+\mathrm{F}-$, podoplanin-positive/FGF1-negative cases; $\mathrm{P}-\mathrm{F}+$, podoplanin-negative/FGF1-positive cases; P-F-, podoplanin-negative/FGF1-negative cases; TNM, tumor node metastasis.

correlation between podoplanin and FGF1 expression in tumor cells $(\mathrm{R}=0.591, \mathrm{P}<0.0001$; Fig. 2C). The cohort of patients was classified into 4 groups according to the expression of podoplanin and FGF1 in the same patient. As presented in Table I, $60.98 \%(50 / 82)$ had positive expression of podoplanin and FGF1 (P+F+), 12.20\% (10/82) had positive expression of podoplanin but negative expression of FGF1 (P+F-), 9.76\% (8/82) patients had positive expression of FGF1 but negative expression of podoplanin $(\mathrm{P}-\mathrm{F}+)$, and $17.07 \%(14 / 82)$ patients had negative expression of podoplanin and FGF1 (P-F-). The $\mathrm{P}+\mathrm{F}+$ group was significantly associated with larger primary tumor size $(\mathrm{P}=0.042)$ and advanced TNM stages $(\mathrm{P}=0.042)$ compared with the P-F- group. Whereas, no clinicopathological factors were demonstrated to be associated with the P+F- or P-F+ groups when compared with the P-F- group (Table I).

Co-expression of podoplanin and FGF1 are associated with intratumoral MVD in primary lung SQCC tissues.
Given the reported effects of podoplanin and FGF1 on lymphangiogenesis and angiogenesis, the present study evaluated the association between their separate expression, and MVD and LMVD in samples. The intratumoral MVD was significantly lower in podoplanin-negative cases compared with podoplanin-positive cases, and the same was observed for FGF1 (data not shown). However, there was no significant difference in intratumoral or peritumoral LMVD between the podoplanin-positive group and podoplanin-negative group, as was also the case for FGF1 (data not shown). The association between co-expression of podoplanin and FGF1, and intratumoral MVD was also investigated; intratumoral MVD (observed by CD34 staining) was demonstrated to be associated with the co-expression of podoplanin and FGF1. The mean MVD was 33.32 \pm 8.01 in $\mathrm{P}+\mathrm{F}+$ specimens, $28.72 \pm 5.93$ in $\mathrm{P}+\mathrm{F}-$ specimens, $29.35 \pm 9.30$ in $\mathrm{P}-\mathrm{F}+$ specimens and 26.64 \pm 6.22 in P-F- specimens. The MVD in the $\mathrm{P}+\mathrm{F}+$ group was significantly higher compared with the 
A

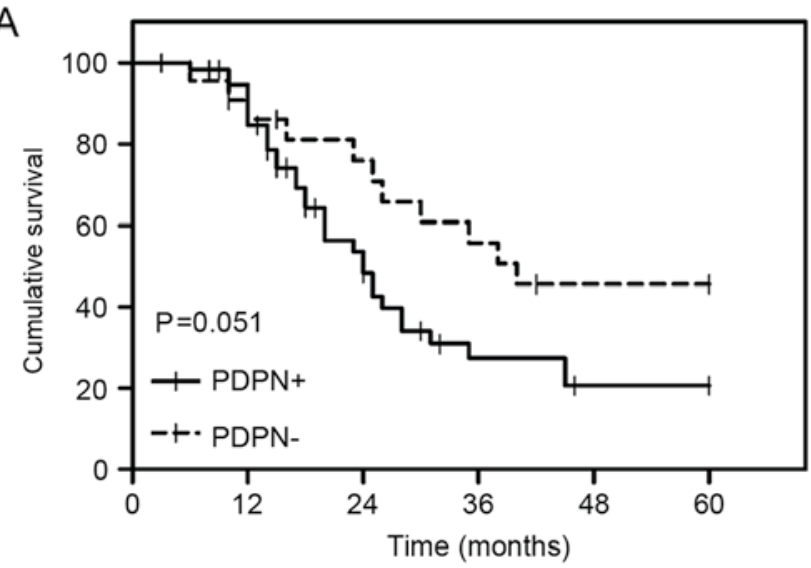

B

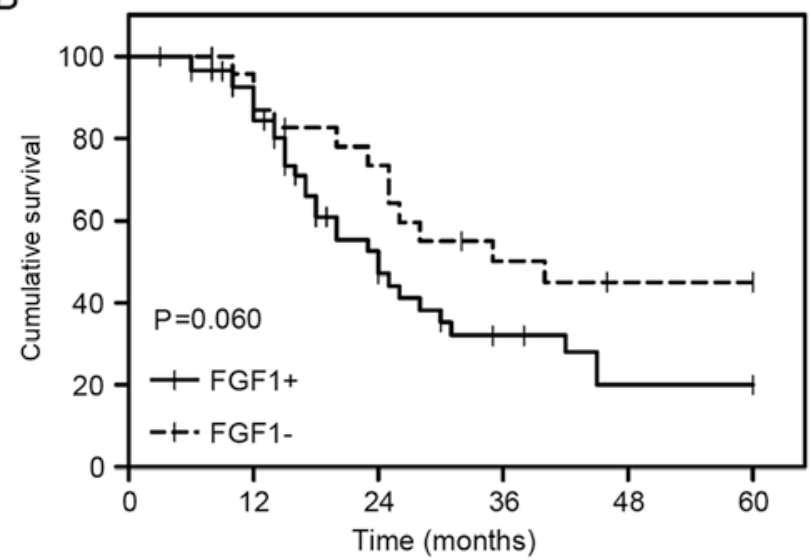

C

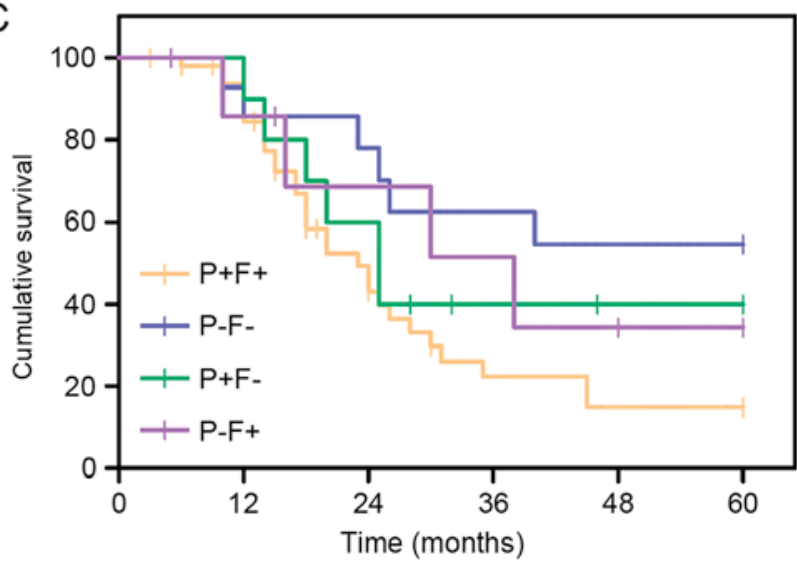

Figure 3. Associations between the expression of podoplanin and FGF1, and overall survival of patients with lung squamous cell carcinoma. (A) Differences in the overall survival rates of podoplanin-positive and podoplanin-negative patients were analyzed. (B) Differences in the overall survival rates of FGF1-positive and FGF1-negative patients were analyzed. (C) Differences in the overall survival rates among $\mathrm{P}+\mathrm{F}+, \mathrm{P}+\mathrm{F}-, \mathrm{P}-\mathrm{F}+$ and $\mathrm{P}-\mathrm{F}$ - patients were analyzed. $\mathrm{P}+\mathrm{F}+$ had a significantly lower survival rate compared with the P-F- group. PDPN, podoplanin; FGF1, fibroblast growth factor $1 ; \mathrm{P}+\mathrm{F}+$, podoplanin-positive/FGF1-positive; $\mathrm{P}+\mathrm{F}-$, podoplanin-positive/FGF1-negative; $\mathrm{P}-\mathrm{F}+$, podoplanin-negative/FGF1-positive; P-F-, podoplanin-negative/FGF1-negative.
P-F- group (Fig. 2D). No significant difference was identified between other pairwise comparisons.

Association between podoplanin/FGF1 co-expression and overall survival of lung SQCC patients. The prognostic value of podoplanin and FGF1 expression in the samples was assessed. The was a difference in the overall survival rate between podoplanin-positive and podoplanin-negative, however, the $\mathrm{P}$-value was marginally above that considered to be significant $(\mathrm{P}=0.051$; Fig. $3 \mathrm{~A})$, which was also observed for FGF1 ( $\mathrm{P}=0.060$; Fig. 3B). Subsequently, the prognostic significance of co-expression of podoplanin and FGF1 for lung SQCC patients was investigated. Survival analyses demonstrated that the $\mathrm{P}+\mathrm{F}+$ group exhibited a significantly lower survival rate compared with the P-F- group $(\mathrm{P}=0.017$; Fig. 3C). No significant differences were observed between all other pairwise comparisons.

Podoplanin regulates the expression of FGF1 and angiogenesis in vitro. Based on the proven effect of podoplanin on VEGFC and endothelin-1 (14,15), which are established lymphangiogenic and angiogenic factors, we hypothesized that the clinicopathological association between podoplanin and FGF1 may be due to the regulatory effect of podoplanin on FGF1 expression. To investigate whether podoplanin regulates the expression of FGF1 in vitro, the present study used siRNA transfection to transiently suppress the expression of podoplanin in the NCI-H226 lung SQCC cell line (Fig. 4A and B). Subsequently, the expression level of FGF1 in podoplanin-knockdown (podoplanin-KD) and NC NCI-H226 cells was investigated. RT-qPCR and western blotting revealed that the expression level of FGF1 mRNA and protein were reduced in podoplanin-KD NCI-H226 cells compared with NC NCI-H226 cells (Fig. 4B and C). As FGF1 is a secreted protein, the level of FGF1 protein in cell culture supernatants was also determined. Compared with NC NCI-H226 cells, podoplanin-KD cells secreted a reduced amount of FGF1 (Fig. 4D).

Considering the association between podoplanin expression and intratumoral MVD, the present study further investigated whether podoplanin affected angiogenesis in vitro. As presented in Fig. 4E and F, HUVECs cultured with tumor cell-conditioned medium from podoplanin-KD NCI-H226 cells exhibited reduced tube formation and a reduced number of branch points compared with the control group.

\section{Discussion}

Podoplanin is upregulated in tumor cells in several cancer types and has been identified as a useful prognostic biomarker to determine the malignancy of tumors $(8,21,22)$. Anti-podoplanin monoclonal antibodies have been validated to suppress the growth and hematogenous metastasis of podoplanin-expressing tumors (23). Researchers have identified several potential pathways that may be invoked by podoplanin to promote tumor progression, including the promotion of 
A
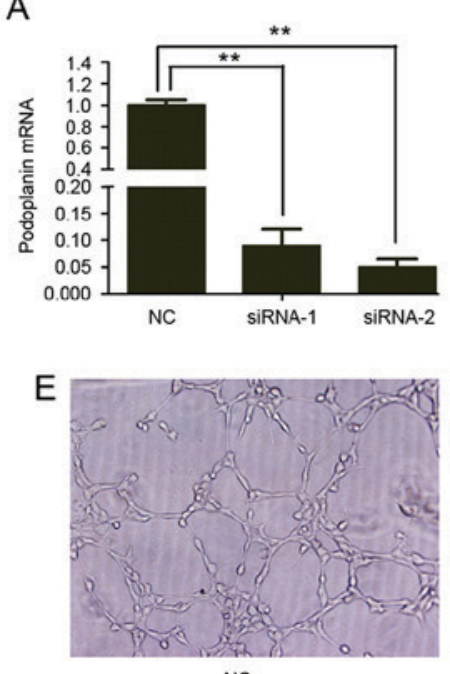

NC
B

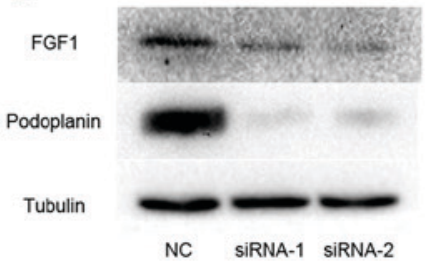

C

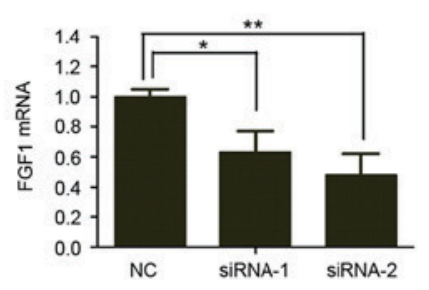

D

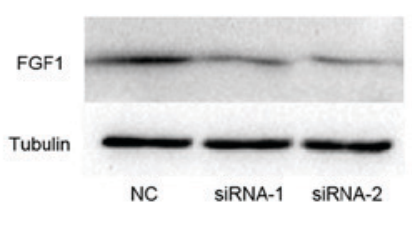

Figure 4. Podoplanin regulated FGF1 expression and angiogenesis in vitro. Confirmation of podoplanin knockdown by siRNA in NCI-H226 cells using (A) RT-qPCR. (B) Western blot analysis showed podoplanin knockdown and FGF1 expression in NCI-H226 cells. (C) RT-qPCR was used to demonstrate the effect of podoplanin knockdown on FGF1 mRNA. (D) Cell supernatants were subjected to western blotting for FGF1. Cell counts and $\alpha$-tubulin of the lysates of the same batch cells was used as loading control. (E) Effect of podoplanin on the formation of tube-like structures by human umbilical vein endothelial cells. Original magnification, x200. (F) Comparison of the numbers of branch points of endothelial tubes in podoplanin-knockdown and NC groups. Each experiment was repeated at least three times. ${ }^{*} \mathrm{P}<0.05$ and ${ }^{* *} \mathrm{P}<0.01$. FGF1, fibroblast growth factor 1 ; siRNA, small interfering RNA; RT-qPCR, reverse transcription-quantitative polymerase chain reaction; NC, negative control.

epithelial-to-mesenchymal transition (EMT) (24), inducing collective cell migration (25), triggering platelet activation and aggregation (26-28) and enhancing lymphangiogenesis (15). The present study demonstrated that podoplanin was co-expressed with FGF1 in cancer cells and was associated with intratumoral MVD in primary lung SQCC tissues. In addition, knockdown of podoplanin downregulated the expression of FGF1 and decreased the formation of tubular networks by HUVECs in vitro. Co-expression of podoplanin and FGF1 was significantly associated with larger primary tumor size, advanced TNM stage, higher intratumoral MVD and worse overall survival. Combined, the results indicate that podoplanin was implicated in tumor progression in lung SQCC when co-expressed with FGF1.

Podoplanin $^{-/}$mice exhibit systemic edema due to aplasia of lymphatic vessels during fetal development and neonatal death due to respiratory failure $(29,30)$, indicating an important role of podoplanin in normal lymphatic vessel development. Furthermore, a previous study demonstrated podoplanin expression was significantly decreased in preeclamptic placental tissues compared with normotensive placental controls, indicating that podoplanin may support fetal vessel angiogenesis during placental development (31). However, several studies concerned with the involvement of podoplanin in lymphangiogenesis/angiogenesis in malignant tumors have produced inconsistent results in different experimental models. Research by Cueni et al (15) demonstrated that podoplanin upregulated endothelin-1 to enhance lymphangiogenesis and metastasis to regional lymph nodes in breast carcinoma xenografts (15). In addition, Suzuki et al (14) reported that podoplanin attenuated lymphogenous metastasis and lymphangiogenesis by downregulating VEGFC in a lung squamous cancer cell line, with no impact observed on angiogenesis (14).
The present study did not demonstrate a significant association between the expression of podoplanin in cancer cells and intratumoral/peritumoral LMVD in human lung SQCC tissues. This inconsistency may be due to the specificity of different cancer types. As demonstrated by previous studies, podoplanin does not exert the same function in all cell types. For example, although podoplanin upregulates RhoA activity and induces EMT in MDCK cells (24), it attenuated RhoA activity and did not induce EMT in a breast carcinoma cell line (25). In fact, Suzuki et al (14) demonstrated that podoplanin decreased the area and perimeter of lymphatic vessels, however, it had no significant effect on the number of lymphatic vessels in an animal model (14), which was consistent with the results of the present study.

The present study, in contrast to the results of Suzuki et al (14), demonstrated that podoplanin was associated with intratumoral MVD and was co-expressed with FGF1 in primary lung SQCC tissues. Similarly, Pula et al (16) observed that podoplanin expression in CAFs was positively correlated with cancer cell VEGFC expression and intratumoral MVD in invasive ductal carcinoma of breast by immunohistochemistry (16). These experimental results indicated that podoplanin may participate in tumor angiogenesis mediated by specific angiogenic growth factors. VEGFC, a regulator of lymphangiogenesis, also contributes to angiogenesis (32). The present study validated the regulation FGF1 expression and angiogenesis by podoplanin in vitro and hypothesized that podoplanin may exert angiogenic action through FGF1, an established angiogenic growth factor. However, the current study did not demonstrate any direct evidence proving the association between the podoplanin-FGF1 axis and podoplanin-dependent angiogenesis, which requires further research. Furthermore, it was previously reported that FGF1 
induced the expression of CD44s (33), and podoplanin interacted with CD44s to drive directional cell migration in tumor cells (34). Therefore, the association between podoplanin and FGF1 may not be confined to tumor angiogenesis, they may also work together to regulate tumor cell motility.

Consistent with the results of our previous study, FGF1 expression was detected at the periphery of cell nests in certain lung SQCC specimens, which was similar to podoplanin and CD44, which are candidate cancer stem cell markers (11). FGF1 has been reported to be upregulated in cancer stem cells of small cell lung cancer (35) and it contributes to sustaining neural stem cell growth and self-renewal capacity in glioblastoma (36). Therefore, FGF1 may be a candidate cancer stem cell marker, and the double-positive expression of podoplanin and FGF1 may have potential as a more accurate screening method for the detection of cancer stem cells. However, the current study did not investigate the molecular pathway by which podoplanin regulated the expression of FGF1, which requires further investigation.

According to the survival analysis performed in the present study, the overall survival was lower in patients with podoplanin-positive lung SQCC compared with patients with podoplanin-negative lung SQCC, although not to a significant extent. In fact, previous studies have produced controversial results regarding the prognostic role of podoplanin in lung SQCC. Kadota et al (8) reported tumor cell podoplanin immunoreactivity to be a significant indicator of poor prognosis in NSCLC patients, particularly in lung SQCC patients, which was consistent with the results of the present study. By contrast, other studies have demonstrated that low podoplanin expression in tumor cells predicts poor prognosis in lung SQCC $(11,37,38)$. The reason behind the conflicting results remains unclear; differences in the way that a positive or high expression of podoplanin is defined across different studies may be one of the reasons behind this discrepancy. However, a significant correlation has been observed between the pattern of podoplanin expression and tumor grade, that is, diffuse infiltrating positivity was detected in undifferentiated tumors and a peripheral pattern in well-differentiated ones $(22,39)$. In the current study, a higher proportion of lung SQCC patients exhibited positive reactions in the majority of the cancer cell nest than previously reported. This may partially explain the disagreement between results of previous studies. Furthermore, the present study demonstrated that cases with double-positive podoplanin and FGF1 staining had significantly shorter survival times compared with those with double negative staining, indicating that integrated assessment of podoplanin and FGF1 may more accurately predict the prognosis of lung SQCC patients compared with either of them alone.

In conclusion, the present study confirmed that podoplanin was co-expressed with FGF1 in lung SQCC and that co-expression was associated with higher intratumoral MVD and poor prognosis of patients with lung SQCC. In vitro experiments demonstrated that podoplanin regulated the FGF1 expression and tube formation of HUVECs. The results indicate that podoplanin may participate in angiogenesis and tumor progression mediated by FGF1 in lung SQCC. Further investigated is necessary to establish the mechanism by which podoplanin regulates FGF1 expression and the synergistic effect mechanism of the two molecules.

\section{Acknowledgements}

This study was supported by the Project of Jinan Science and Technology Plan (grant no. 201101112). We thank Dong Zhao and Ling Wang of Department of Pathology, Jinan Central Hospital, Shandong University (Jinan, China) for assisting with the collection of NSCLC samples and preparing tissue sections. We acknowledge Dr Huiping Liu of the Department of Pathology, Jinan Central Hospital, Shandong University for quantifying immunohistochemical sections.

\section{References}

1. World Health Organization Media Centre. Cancer. Fact sheet no. 297. http://www.who.int/mediacentre/factsheets/fs297/en/ Accessed February, 2017.

2. Herbst RS, Heymach JV and Lippman SM: Lung Cancer. N Engl J Med 359: 1367-1380, 2008.

3. Pao W, Miller V, Zakowski M, Doherty J, Politi K, Sarkaria I, Singh B, Heelan R, Rusch V, Fulton L, et al: EGF receptor gene mutations are common in lung cancers from 'never smokers' and are associated with sensitivity of tumors to gefitinib and erlotinib. Proc Natl Acad Sci USA 101: 13306-13311, 2004.

4. Kwak EL, Bang YJ, Camidge DR, Shaw AT, Solomon B, Maki RG, Ou SH, Dezube BJ, Jänne PA, Costa DB, et al: Anaplastic lymphoma kinase inhibition in non-small-cell lung cancer. N Engl J Med 363: 1693-1703, 2010.

5. Daraselia N, Wang Y, Budoff A, Lituev A, Potapova O, Vansant G, Monforte J, Mazo I and Ossovskaya VS: Molecular signature and pathway analysis of human primary squamous and adenocarcinoma lung cancers. Am J Cancer Res 2: 93-103, 2012.

6. Heist RS, Sequist LV and Engelman JA: Genetic changes in squamous cell lung cancer: A review. J Thorac Oncol 7: 924-933, 2012.

7. Kahn HJ and Marks A: A new monoclonal antibody, D2-40, for detection of lymphatic invasion in primary tumors. Lab Invest 82: 1255-1257, 2002

8. Kadota K, Huang CL, Liu D, Nakashima N, Yokomise H, Ueno M and Haba R: The clinical significance of the tumor cell D2-40 immunoreactivity in non-small cell lung cancer. Lung Cancer 70: 88-93, 2010.

9. Atsumi N, Ishii G, Kojima M, Sanada M, Fujii S and Ochiai A: Podoplanin, a novel marker of tumor-initiating cells in human squamous cell carcinoma A431. Biochem Biophys Res Commun 373: 36-41, 2008.

10. Li J, Wei Z, Li H, Dang Q, Zhang Z, Wang L, Gao W, Zhang P, Yang D, Liu J, et al: Clinicopathological significance of fibroblast growth factor 1 in non-small cell lung cancer. Hum Pathol 46: 1821-1828, 2015.

11. Shimada Y, Ishii G, Nagai K, Atsumi N, Fujii S, Yamada A, Yamane Y, Hishida T, Nishimura M, Yoshida J, et al: Expression of podoplanin, CD44, and p63 in squamous cell carcinoma of the lung. Cancer Sci 100: 2054-2059, 2009.

12. Presta M,Dell'Era P, Mitola S, Moroni E, Ronca R and Rusnati M: Fibroblast growth factor/fibroblast growth factor receptor system in angiogenesis. Cytokine Growth Factor Rev 16: 159-178, 2005.

13. Shin JW, Min M, Larrieu-Lahargue F, Canron X, Kunstfeld R, Nguyen L, Henderson JE, Bikfalvi A, Detmar M and Hong YK: Prox1 promotes lineage-specific expression of fibroblast growth factor (FGF) receptor-3 in lymphatic endothelium: A role for FGF signaling in lymphangiogenesis. Mol Biol Cell 17: 576-584, 2006.

14. Suzuki H, Onimaru M, Yonemitsu Y, Maehara Y, Nakamura S and Sueishi K: Podoplanin in cancer cells is experimentally able to attenuate prolymphangiogenic and lymphogenous metastatic potentials of lung squamoid cancer cells. Mol Cancer 9: 287,2010

15. Cueni LN, Hegyi I, Shin JW, Albinger-Hegyi A, Gruber S, Kunstfeld R, Moch H and Detmar M: Tumor lymphangiogenesis and metastasis to lymph nodes induced by cancer cell expression of podoplanin. Am J Pathol 177: 1004-1016, 2010.

16. Pula B, Wojnar A, Witkiewicz W, Dziegiel P and PodhorskaOkolow M: Podoplanin expression in cancer-associated fibroblasts correlates with VEGF-C expression in cancer cells of invasive ductal breast carcinoma. Neoplasma 60: 516-524, 2013.

17. Sobin LH GM and Wittekind C (eds): UICC TNM Classification of Malignant Tumours. 7th edition. Wiley-Liss, New York, NY, 2009. 
18. Livak KJ and Schmittgen TD: Analysis of relative gene expression data using real-time quantitative PCR and the 2(-Delta Delta C(T)) Method. Methods 25: 402-408, 2001.

19. Engels K, Knauer SK, Metzler D, Simf C, Struschka O, Bier C, Mann W, Kovács AF and Stauber RH: Dynamic intracellular survivin in oral squamous cell carcinoma: Underlying molecular mechanism and potential as an early prognostic marker. J Pathol 211: 532-540, 2007.

20. Weidner N, Semple JP, Welch WR and Folkman J: Tumor angiogenesis and metastasis-correlation in invasive breast carcinoma. N Engl J Med 324: 1-8, 1991.

21. Yuan P, Temam S, El-Naggar A, Zhou X, Liu DD, Lee JJ and Mao L: Overexpression of podoplanin in oral cancer and its association with poor clinical outcome. Cancer 107: 563-569, 2006

22. Minardi D, d'Anzeo G, Lucarini G, Filosa A, Zizzi A Simonetti O, Polito M Jr, Offidani AM, Di Primio R, Montironi R, Muzzonigro G: D2-40 immunoreactivity in penile squamous cell carcinoma: A marker of aggressiveness. Hum Pathol 42: 1596-1602, 2011

23. Nakazawa Y, Takagi S, Sato S, Oh-hara T, Koike S, Takami M, Arai $\mathrm{H}$ and Fujita N: Prevention of hematogenous metastasis by neutralizing mice and its chimeric anti-Aggrus/podoplanin antibodies. Cancer Sci 102: 2051-2057, 2011.

24. Martín-Villar E, Megías D, Castel S, Yurrita MM, Vilaró S and Quintanilla M: Podoplanin binds ERM proteins to activate RhoA and promote epithelial-mesenchymal transition. J Cell Sci 119: 4541-4553, 2006.

25. Wicki A, Lehembre F, Wick N, Hantusch B, Kerjaschki D and Christofori G: Tumor invasion in the absence of epithelial-mesenchymal transition: Podoplanin-mediated remodeling of the actin cytoskeleton. Cancer Cell 9: 261-272, 2006.

26. Kunita A, Kashima TG, Morishita Y, Fukayama M, Kato Y, Tsuruo $\mathrm{T}$ and Fujita N: The platelet aggregation-inducing factor aggrus/podoplanin promotes pulmonary metastasis. Am J Pathol 170: 1337-1347, 2007.

27. Suzuki-Inoue K, Kato Y, Inoue O, Kaneko MK, Mishima K, Yatomi Y, Yamazaki Y, Narimatsu H and Ozaki Y: Involvement of the Snake Toxin Receptor CLEC-2, in Podoplanin-mediated platelet activation, by cancer cells. J Biol Chem 282: 25993-26001, 2007.

28. Nakazawa Y, Sato S, Naito M, Kato Y, Mishima K, Arai H, Tsuruo T and Fujita N: Tetraspanin family member CD9 inhibits Aggrus/podoplanin-induced platelet aggregation and suppresses pulmonary metastasis. Blood 112: 1730-1739, 2008.

29. Ramirez MI, Millien G, Hinds A, Cao Y, Seldin DC and Williams MC: T1alpha, a lung type I cell differentiation gene, is required for normal lung cell proliferation and alveolus formation at birth. Dev Biol 256: 61-72,2003.
30. Schacht V, Ramirez MI, Hong YK, Hirakawa S, Feng D, Harvey N, Williams M, Dvorak AM, Dvorak HF, Oliver G and Detmar M: T1alpha/podoplanin deficiency disrupts normal lymphatic vasculature formation and causes lymphedema. EMBO J 22: 3546-3556, 2003.

31. Wang Y, Sun J, Gu Y, Zhao S, Groome LJ and Alexander JS D2-40/podoplanin expression in the human placenta. Placenta 32: 27-32, 2011.

32. Nagai $\mathrm{N}$ and Minami T: Emerging role of VEGFC in pathological angiogenesis. EBioMedicine 2: 1588-1589, 2015.

33. Palen KA, Jing W, Weber JJ, Tilkens SB, Chan AM, Johnson BD and Gershan JA: Separation and characterization of epithelial and mesenchymal-like murine mammary tumor cells reveals epithelial cell differentiation plasticity and enhanced tumorigenicity of epithelial-enriched tumor cells. Cancer Microenviron 6: 79-89, 2013.

34. Martin-Villar E, Fernández-Muãoz B, Parsons M, Yurrita MM, Megias D, Pérez-Gómez E, Jones GE and Quintanilla M: Podoplanin associates with CD44 to promote directional cell migration. Mol Biol Cell 21: 4387-4399, 2010.

35. Salcido CD, Larochelle A, Taylor BJ, Dunbar CE and Varticovski L: Molecular characterisation of side population cells with cancer stem cell-like characteristics in small-cell lung cancer. Br J Cancer 102: 1636-1644, 2010.

36. Hsu YC, Lee DC, Chen SL, Liao WC, Lin JW, Chiu WT and Chiu IM: Brain-specific 1B promoter of FGF1 gene facilitates the isolation of neural stem/progenitor cells with self-renewal and multipotent capacities. Dev Dyn 238: 302-314, 2009.

37. Ito T, Ishii G, Nagai K, Nagano T, Kojika M, Murata Y, Atsumi N, Nishiwaki Y, Miyazaki E, Kumamoto T and Ochiai A: Low podoplanin expression of tumor cells predicts poor prognosis in pathological stage IB squamous cell carcinoma of the lung, tissue microarray analysis of 136 patients using 24 antibodies. Lung Cancer 63: 418-424, 2009.

38. Suzuki H, Onimaru M, Koga T, Takeshita M, Yano T, Maehara Y, Nakamura S and Sueishi K: High podoplanin expression in cancer cells predicts lower incidence of nodal metastasis in patients with lung squamous cell carcinoma. Pathol Res Pract 207: 111-115, 2011.

39. Ikoma Y, Kijima H, Masuda R, Tanaka M, Inokuchi S and Iwazaki M: Podoplanin expression is correlated with the prognosis of lung squamous cell carcinoma. Biomed Res 36: 393-402, 2015. 